\title{
Dosimetric Comparison of Volumetric-Modulated Arc Therapy and Dynamic Conformal Arc Therapy in Three- fraction Single-isocenter Stereotactic Radiosurgery for Multiple Brain Metastases
}

\author{
(1) Görkem TÜRKKAN, ${ }^{1,2}$ (D) Nazlı BiLici, ${ }^{2}$ (D) Hüseyin SERTEL, ${ }^{2}$ (D) Büşra TAVLI, ${ }^{2}$ (1) Müge ÖZKIRIM, ${ }^{2}$ \\ (1) Merdan FAYDA ${ }^{1,2}$
}

'Department of Radiation Oncology, Istinye University Faculty of Medicine, Istanbul-Turkey

${ }^{2}$ Department of Radiation Oncology, Liv Hospital Ulus, Istanbul-Turkey

\section{OBJECTIVE}

The objective of the study was to compare single-isocenter volumetric-modulated arc therapy (VMAT) and dynamic conformal arc therapy (DCAT) techniques in patients who received multifraction stereotactic radiosurgery (SRS) for multiple brain metastases.

\section{METHODS}

Twenty-one patients who were treated with a radiation dose of $27 \mathrm{~Gy}$ in three fractions were replanned. Both VMAT and DCAT plans with single isocenter were obtained for each patient. Plan quality indices, cumulative monitor unit $(\mathrm{MU})$ values, maximum dose for organs at risk, and mean dose, V19.6 $\mathrm{Gy}^{\prime}$ and V23. $1_{\mathrm{Gy}}$ for healthy brain tissue were compared.

\section{RESULTS}

The conformity index $(\mathrm{p}=0.0002)$, gradient index $(\mathrm{p}=0.003)$, maximum dose for brainstem $(\mathrm{p}=0.016)$ and mean dose $(\mathrm{p}=0.00007), \mathrm{V} 19.6_{\mathrm{Gy}}(\mathrm{p}=0.00006)$, and $\mathrm{V} 23.1_{\mathrm{Gy}}(\mathrm{p}=0.00006)$ values for healthy brain tissue were significantly superior in the VMAT technique, compared to DCAT technique. In addition, a trend toward significance for achieving lower maximum dose value to the optic nerves and/or pathway was observed with VMAT $(\mathrm{p}=0.073)$. DCAT provided significantly lower MU values (3097.44 vs. 1479.09; $\mathrm{p}=0.00006$ ).

\section{CONCLUSION}

VMAT was able to provide better target conformity and lower risk of brain radionecrosis at least dosimetrically in multifraction SRS for patients with multiple brain metastases. DCAT may be chosen in patients with relatively poor performance status or low tolerance to long-lasting radiotherapy sessions.

Keywords: Dosimetric comparison; dynamic conformal arc therapy; multiple brain metastases; stereotactic radiotherapy; volumetric-modulated arc therapy.

Copyright $\odot$ 2022, Turkish Society for Radiation Oncology

\section{Introduction}

Stereotactic radiosurgery (SRS) is increasingly preferred over whole-brain radiotherapy (WBRT) and widely accepted as the standard of care for patients with multiple brain metastases. Brain SRS not only improves local control but also allows to avoid the potential side effects of WBRT by substantially reducing 
doses to critical organs. Brain SRS can be used for up to 10 metastases, depending on many factors including the maximum diameter, total volume, and number of the lesions.[1,2] Non-inferior brain SRS outcomes were also shown in patients with more than 10 metastases, compared to those with 2-9 metastases.[3]

Radiation necrosis is a dose-limiting toxicity of brain SRS. The previous studies have shown the relationship between the occurrence of radionecrosis after brain SRS and the volume of healthy brain tissue exposed to both high and low doses of radiation. [4,5] On the other hand, fractionated SRS is suggested as a promising tool that may reduce the risk of radionecrosis and provide similar local control results to singlefraction SRS.[6,7] Three-fraction SRS is one of the most commonly used radiotherapy schemes for brain SRS. Limitations for healthy brain tissue volumes receiving doses of $\geq 19.6 \mathrm{~Gy}$ (non-planning target volume [PTV] brain V19.6 ${ }_{\mathrm{Gy}}$ ) and $\geq 23.1 \mathrm{~Gy}$ (non-PTV brain $\mathrm{V} 23.1_{\mathrm{Gy}}$ ) are recently proposed for the risk estimation of brain radionecrosis after three-fraction SRS.[8]

At present, linear accelerator (LINAC)-based SRS is commonly used worldwide and represents an important part of radiotherapy applications for brain metastases. The integration of modern radiotherapy techniques, such as intensity-modulated radiotherapy (IMRT) and volumetric-modulated arc therapy (VMAT) into clinical practice, has led to significant improvements in LINAC-based SRS planning. In recent years, there has been an increased interest in using dynamic conformal arc therapy (DCAT) technique in stereotactic radiotherapy. DCAT can be used for brain SRS of single and even multiple brain metastases.[9] DCAT uses variable dose rate (VRD) and segment shape optimization (SSO). SSO provides beam modulation for better dose conformity and normal tissue sparing, and thus supports Monaco treatment planning system (TPS) to be able to achieve similar plan quality to VMAT. With the combination of SSO and VRD, DCAT can reduce cumulative MU values and enable quicker plans.[10] VMAT, on the other hand, does require much more MUs to deliver the same total dose, due to the increased modulation.[11] The potential contributions of these advances in treatment planning techniques to radiotherapy practice are always worth exploring.

This study aimed to compare single-isocenter VMAT and DCAT techniques for multifraction SRS for multiple brain metastases, in terms of healthy brain tissue sparing, critical organ doses, quality indices, and cumulative monitor unit (MU) values. In this context, there are very few studies in the literature and nearly all of these studies include patients treated with singlefraction SRS. $[9,12]$ To the best of our knowledge, this is the first study comparing VMAT and DCAT techniques in patients with multiple brain metastases who were treated with single-isocenter three-fraction SRS.

\section{Materials and Methods}

The study was conducted as a retrospective dosimetric study. The Institutional Ethics Committee approved the study protocol.

\section{Patient Selection}

Patients with 2-10 brain metastases treated with fractionated brain SRS in Elekta Versa HD linear accelerator (Elekta, Crawley, UK) at our institution were included in this study. The prescribed dose for each target was $27 \mathrm{~Gy}$ in three fractions.

Patients with multiple brain metastases who were treated with radiation dose different from 27 Gy in three fractions were excluded from the study. In addition, none of the patients had single brain metastasis, more than 10 brain metastases, or a history of brain surgery.

\section{Immobilization, Contouring, and Prescription}

Each patient was immobilized using a thermoplastic mask in the supine position and scanned with computed tomography (CT) from the vertex to the base of skull with a scan thickness of $1.25 \mathrm{~mm}$. No contrast agent was used. The obtained planning CT images were appropriately fused with the contrast-enhanced brain magnetic resonance (MR) images of the patients.

The brain, brainstem, optic nerves/chiasm, lenses, eyes, and cohleas were contoured as organs at risk (OARs) in accordance with the European Particle Therapy Network consensus-based contouring atlas. [13] The gross tumor volumes (GTVs) were delineated on fused MR/CT images. The PTV was defined as GTV plus $2 \mathrm{~mm}$ margin to allow for inter-, intra-fractional, and set-up errors. The volume of the healthy brain tissue (non-PTV brain) was defined as the volume of the brain minus PTV.

\section{Treatment Planning and Templates}

Radiotherapy plans of the patients who were treated with multifraction brain SRS were replanned by the same medical physicist in the Monaco TPS version 5.0 (Elekta, Crawley, UK). In this way, both VMAT and DCAT plans were obtained for each patient. Flattening 
filter-free $6 \mathrm{MV}$ (1400 MU/min) photon beams were used in all plans.

Both in VMAT and DCAT plans, the isocenter was placed at the geometric center of the PTVs. Same gantry and couch angles were used for each plan, and they were chosen wisely depending on the localization of lesions by the same medical physicist. Both DCAT and VMAT plans were based on five non-coplanar arcs. These partial non-coplanar arcs had an arc length of $120^{\circ}$, regarding gantry rotation. The couch angles were $10^{\circ}, 45^{\circ}, 90^{\circ}, 315^{\circ}$, and $345^{\circ}$; and their corresponding gantry start angles were $200^{\circ}$ clockwise (CW), $320^{\circ}$ counter-clockwise (CCW), $200^{\circ} \mathrm{CW}, 160^{\circ} \mathrm{CCW}$, and $40^{\circ} \mathrm{CW}$, respectively. Due to the different localization of the metastases, collimator angles were different for each plan. All plans were calculated using Monte Carlo algorithm. The treatment couch, immobilization devices, and thermoplastic mask were included in the dose calculation. Dose distributions were calculated using the $2 \mathrm{~mm}$ grid size, and a Monte Carlo dose calculation uncertainty of $1 \%$ per calculation was used. SSO was used in all DCAT plans to be able to reach the higher conformity potential of VMAT. Task group 101 dose constraints for three fractions were considered for dose limitations for OAR.[14]

\section{Metrics for Plan Comparison}

The plan quality indices including conformity index (CI), gradient index (GI), and dose homogeneity index (DHI) were calculated as previously proposed by Radiation Therapy Oncology Group (RTOG) and Paddick and Lippitz, $[15,16]$ and the calculation methods are as follows:

$\mathrm{CI}_{\text {RTOG }}=$ The prescription isodose volume/Target volume $\mathrm{DHI}_{\mathrm{RTOG}}=$ The maximum dose in the target/The prescription dose

$\mathrm{GI}_{\text {Paddick }}=$ The volume covered by half of the prescribed isodose/Target volume
Mean dose $\left(\mathrm{D}_{\text {mean }}\right), \mathrm{V} 19.6_{\mathrm{Gy}}$ and V23.1 $1_{\mathrm{Gy}}$ for nonPTV brain, maximum dose $\left(\mathrm{D}_{\max }\right)$ to the OARs (brainstem, lenses, cochlea, and optic nerves/chiasm), and cumulative MU values were extracted from the TPS for each plan. The values of these plan quality indices and dose-volume parameters in the VMAT plans were compared with their equivalents in the DCAT plans.

\section{Statistical Analysis}

Statistical analysis was performed using the SPSS software version 22.0 (SPSS Inc., Chicago, IL, USA). The Shapiro-Wilk test was used to assess data normality. Data from the DCAT and VMAT plans were compared using the Wilcoxon signed-rank test and Student's paired t-test, for non-parametric and parametric analyses, respectively. $\mathrm{P}<0.05$ was considered as statistically significant.

\section{Results}

Twenty-one patients with a total of 84 brain metastases were evaluated. The characteristics of the patients are shown in Table 1. All VMAT and DCAT plans were optimized with an identical optimization protocol and the planning goals were achieved with both planning techniques. Target coverage was statistically similar for DCAT and VMAT plans, and at least $97 \%$ of the PTV was covered by the prescription isodose line on each patient. The results for the plan quality indices and the dose-volume parameters in the VMAT and DCAT techniques are shown in Table 2.

Compared with the DCAT technique, $\mathrm{CI}_{\mathrm{RTOG}}$ $(\mathrm{p}=0.0002)$ and $\mathrm{GI}_{\text {Paddick }}(\mathrm{p}=0.003)$ were significantly superior in the VMAT technique. However, no significant difference was found for DHI between two techniques. In addition, VMAT was significantly superior to DCAT in terms of exposed radiation dose of healthy brain tissue including the non-PTV brain V19.6 ${ }_{G y}(p=0.00006)$,

\section{Table 1 Patient characteristics}

\section{Patient characteristic}

Sex

Primary tumor origin

Median age at fractionated SRS (years)

Total number of brain metastases of all patients

Median number of brain metastases per patient

Median planning target volume $\left(\mathrm{cm}^{3}\right)$

Average metastasis diameter (median value, $\mathrm{cm}$ )
Value (n)

Female: 8 , male: 13

Lung: 16, breast: 2, kidney: 1, pancreas: 1, larynx: 1

64 (range: $48-81$ )

84

3 (range: 2-10)

7.00 (range: $1.10-70.59$ )

1.06 (range: $0.44-3.57$ )

SRS: Stereotactic radiosurgery 
Table 2 The comparison of the plan quality indices and dose-volume parameters for VMAT and DCAT techniques

\begin{tabular}{|c|c|c|c|}
\hline Parameter & $\begin{array}{l}\text { VMAT plans; } \\
\text { (no. of superior } \\
\text { VMAT plans) }\end{array}$ & $\begin{array}{l}\text { DCAT plans; } \\
\text { (no. of superior } \\
\text { DCAT plans) }\end{array}$ & $\mathbf{p}$ \\
\hline PTV coverage percentage of the prescription isodose line (mean \pm SD) & $98.1 \pm 1.1 ;(11)$ & $97.8 \pm 0.5(10)$ & 0.565 \\
\hline $\mathrm{Cl}_{\mathrm{RTOG}}($ mean $\pm \mathrm{SD})$ & $1.21 \pm 0.23 ;(19)$ & $1.78 \pm 0.79 ;(2)$ & 0.0002 \\
\hline $\mathrm{Gl}_{\text {Paddick }}($ mean $\pm \mathrm{SD})$ & $5.86 \pm 2.57 ;(19)$ & $8.01 \pm 4.89 ;(2)$ & 0.003 \\
\hline $\mathrm{DHI}_{\mathrm{RTOG}}($ mean $\pm \mathrm{SD})$ & $1.38 \pm 0.94 ;(9)$ & $1.17 \pm 0.04 ;(12)$ & 0.53 \\
\hline Cumulative MU (mean \pm SD) & $3097.44 \pm 934.46 ;(0)$ & $1479.09 \pm 165.31 ;(21)$ & 0.00006 \\
\hline Non-PTV brain $\mathrm{D}_{\text {mean }}($ mean $\pm \mathrm{SD})$ & $3.64 \pm 1.49 ;(20)$ & $4.93 \pm 2.49 ;(1)$ & 0.00007 \\
\hline Non-PTV brain V19.6 $6_{G y}(\mathrm{cc})($ mean \pm SD) & $21.05 \pm 15.37 ;(21)$ & $53.83 \pm 48.73 ;(0)$ & 0.00006 \\
\hline Non-PTV brain V23.1 $1_{\mathrm{Gy}}(\mathrm{cc})($ mean \pm SD) & $12.19 \pm 10.19 ;(21)$ & $33.14 \pm 32.35 ;(0)$ & 0.00006 \\
\hline Brainstem $D_{\max }($ mean $\pm S D)$ & $6.88 \pm 5.11 ;(16)$ & $8.57 \pm 5.97 ;(5)$ & 0.016 \\
\hline Optic nerves/pathway $D_{\max }($ mean $\pm S D)$ & $3.66 \pm 2.45 ;(13)$ & $4.70 \pm 4.07 ;(8)$ & 0.073 \\
\hline Lenses $\mathrm{D}_{\max }($ mean $\pm \mathrm{SD})$ & $1.27 \pm 0.77 ;(9)$ & $1.67 \pm 2.27 ;(12)$ & 0.85 \\
\hline 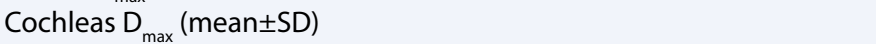 & $4.28 \pm 4.36 ;(14)$ & $4.97 \pm 6.23 ;(7)$ & 0.259 \\
\hline
\end{tabular}

VMAT: Volumetric-modulated arc therapy; DCAT: Dynamic conformal arc therapy; PTV: Planning target volume; Cl: Conformity index; SD: Standard deviation; RTOG: Radiation Therapy Oncology Group; GI: Gradient index; DHI: Dose homogeneity index; MU: Monitor unit; Gy: Gray

V23.1 $1_{\text {Gy }}(\mathrm{p}=0.00006)$, and $\mathrm{D}_{\text {mean }}(\mathrm{p}=0.00007)$ parameters. On the other hand, significantly lower MU values were obtained in the DCAT plans (3097.44 vs. 1479.09; $\mathrm{p}=0.00006)$. A visual comparison of dose distributions in axial, sagittal, and coronal sections of a patient for each planning technique is shown in Figure 1.

The maximum doses to the OARs were also compared. No statistically significant difference was found between two planning techniques, regarding $\mathrm{D}_{\max }$ values to the lenses and cochleas. However, a trend toward significance for achieving lower Dmax value to the optic nerves/pathway was observed with VMAT $(\mathrm{p}=0.073)$. Importantly, the VMAT technique showed statistically significant benefit for achieving lower Dmax values on brainstem $(\mathrm{p}=0.016)$.

\section{Discussion}

The remarkable finding of this study was that the VMAT plans were able to provide better plan conformity, better dose gradient, and steeper dose reduction to the OARs (healthy brain and brainstem), compared to DCAT for multifraction SRS for multiple brain metastases. On the other hand, DCAT plans were superior to VMAT plans in terms of MU values and therefore duration of treatment. DCAT was able to reduce the treatment time by half, when compared to VMAT.

In general, our results were similar to the results in the previous studies comparing VMAT and DCAT in brain SRS.[17-19] However, it should be kept in mind that nearly all of the previous studies include patients treated with single-fraction SRS. As far as we know, the current study represents the first contributions to the literature for three-fraction brain SRS for multiple brain metastases, in this regard.

Obtaining the best possible GI and CI values has been suggested to achieve the optimal stereotactic radiotherapy planning.[15] In this study, the VMAT plans showed superior $\mathrm{CI}_{\mathrm{RTOG}}$ and $\mathrm{GI}_{\text {Paddick }}$ values than the DCAT-based plans. In other words, VMAT can provide more conformal dose distribution with accompanying steeper dose falloff beyond the target volume when compared to DCAT.

Inspired by the recommendations in a recent study of Milano et al.,[8] we evaluated V19.6 ${ }_{\mathrm{Gy}}, \mathrm{V} 23.1_{\mathrm{Gy}}$ and $\mathrm{D}_{\text {mean }}$ parameters for non-PTV brain, for the risk estimation of radionecrosis after brain SRS. The results achieved with VMAT for the aforementioned dosevolume parameters were significantly superior to those obtained with DCAT. In other words, VMAT can lower the risk of brain radionecrosis more than DCAT. Moreover, VMAT could provide lower brainstem $\mathrm{D}_{\max }$ dose when compared to DCAT, and VMAT seems to be one step ahead in patients with metastases close to the brainstem. Considering that patients can live longer and longer with developing treatment methods and that some patients need to be reirradiated due to intracranial recurrence in follow-up, it is very important to keep the radiation dose to which normal brain tissue is exposed as low as possible. Briefly, the use of VMAT should be given priority over DCAT in multifraction brain SRS planning. 


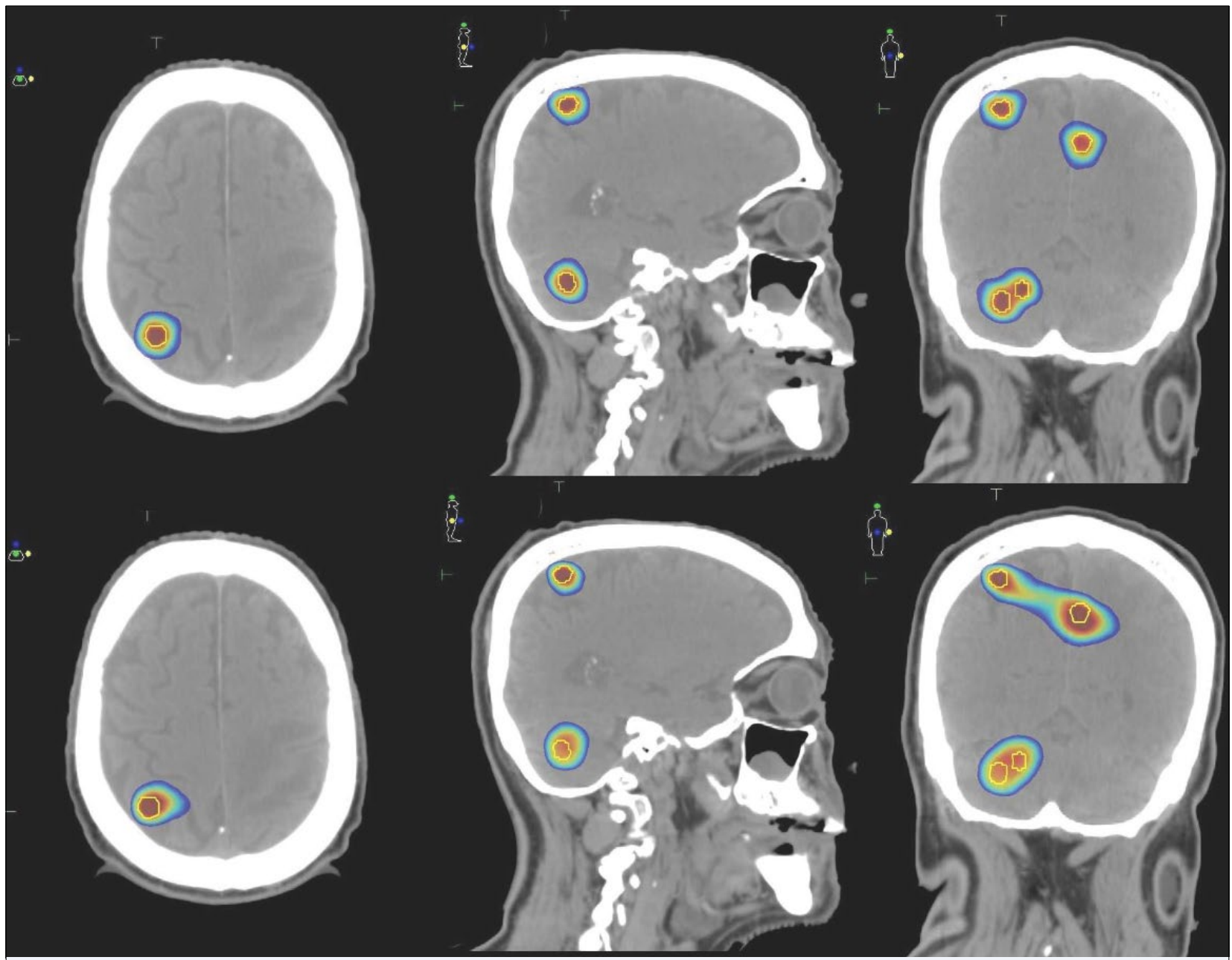

Fig. 1. Dose distributions of a patient with seven brain metastases. Since all lesions cannot be seen in one section, an exemplary image was chosen for each section. The upper three panels and the lower three panels demonstrate axial, coronal, and sagittal sections for the DCAT and the VMAT plans, respectively.

DCAT: Dynamic conformal arc therapy; VMAT: Volumetric-modulated arc therapy.

On the other hand, stereotactic radiotherapy is a time-consuming treatment strategy. In daily practice, it may be of great significance to complete the radiotherapy sessions quickly, especially for patients with relatively poor general condition or low tolerance to long-lasting radiotherapy sessions. The shortening of the treatment time may decrease the undesirable movements, increase the patient's comfort, relieve patient's distress, and thus reduce treatment-related uncertainties in this group of patients.

However, it is clear that many factors such as patient/tumor characteristics, plan quality indices, and the capabilities of the radiotherapy device should be considered to obtain optimal brain SRS plan. While also recognizing all of this, in some selected patients with low tolerance for long-lasting radiotherapy ses- sions, choosing DCAT over VMAT in brain SRS may help clinicians in daily practice, since DCAT could provide similar target coverage while reducing the treatment time by half. In this regard, this study supports the previous studies reporting that DCAT can reduce treatment time in brain SRS, when compared to VMAT.[20,21]

\section{Limitations and Strength of the Study}

There are some limitations to this study. We compared a limited number of plan quality indices and dose-volume parameters, because the optimal parameters are not well-known and/or well-defined. Different stratifications could be applied and different parameters other than those we have compared could be also assessed. In addition, a comparison considering the spheric- 
ity of the target lesions and their distance from each other and critical organs may provide further important data. Due to the nature of the study, it could not be measured whether the dosimetric superiority of VMAT over DCAT was reflected or not in a clinical setting, in terms of toxicity.

The strength of our study is being the first study comparing VMAT and DCAT plans in patients with multiple brain metastases who were treated with single-isocenter three-fraction SRS.

\section{Conclusion}

When these two planning techniques are compared, VMAT seems to be superior to DCAT in multifraction brain SRS for multiple brain metastases in terms of target conformity and the risk of brain radionecrosis. Therefore, VMAT should be given priority over DCAT. DCAT may be chosen only in selected patients with relatively poor performance status or low tolerance to long-lasting radiotherapy sessions.

Peer-review: Externally peer-reviewed.

Conflict of Interest: All authors declared no conflict of interest.

Ethics Committee Approval: The study was approved by the Human Research Ethics Committee of Istinye University (No: 21-70, Date: 23/08/2021).

Financial Support: This study has received no financial support.

Authorship contributions: Concept - G.T., N.B., H.S., B.T., M.Ö., M.F.; Design - G.T., N.B., M.F.; Supervision - G.T., M.F.; Funding - G.T., N.B., H.S., B.T., M.Ö., M.F.; Materials - G.T., N.B., H.S., B.T., M.Ö., M.F.; Data collection and/or processing - G.T., N.B., H.S., B.T., M.Ö.; Data analysis and/ or interpretation - G.T., N.B., H.S.; Literature search - G.T., B.T., M.Ö.; Writing - G.T., N.B.; Critical review - G.T., M.F.

\section{References}

1. Yamamoto M, Serizawa T, Shuto T, Akabane A, Higuchi Y, Kawagishi J, et al. Stereotactic radiosurgery for patients with multiple brain metastases (JLGK0901): A multi-institutional prospective observational study. Lancet Oncol 2014;15(4):387-95.

2. Soffietti R, Abacioglu U, Baumert B, Combs SE, Kinhult S, Kros JM, et al. Diagnosis and treatment of brain metastases from solid tumors: Guidelines from the european association of neuro-oncology (EANO). Neuro Oncol 2017;19(2):162-74.
3. Yamamoto M, Kawabe T, Sato Y, Higuchi Y, Nariai $\mathrm{T}$, Watanabe $\mathrm{S}$, et al. Stereotactic radiosurgery for patients with multiple brain metastases: A casematched study comparing treatment results for patients with 2-9 versus 10 or more tumors. J Neurosurg 2014;121:16-25.

4. Blonigen BJ, Steinmetz RD, Levin L, Lamba MA, Warnick RE, Breneman JC. Irradiated volume as a predictor of brain radionecrosis after linear accelerator stereotactic radiosurgery. Int J Radiat Oncol Biol Phys 2010;77(4):996-1001.

5. Minniti G, Clarke E, Lanzetta G, Osti MF, Trasimeni G, Bozzao A, et al. Stereotactic radiosurgery for brain metastases: Analysis of outcome and risk of brain radionecrosis. Radiat Oncol 2011;6:48.

6. Putz F, Weissmann T, Oft D, Schmidt MA, Roesch J, Siavooshhaghighi $\mathrm{H}$, et al. FSRT vs. SRS in brain metastases-differences in local control and radiation necrosis-a volumetric study. Front Oncol 2020;10:559193.

7. Remick JS, Kowalski E, Khairnar R, Sun K, Morse E, Cherng HR, et al. A multi-center analysis of singlefraction versus hypofractionated stereotactic radiosurgery for the treatment of brain metastasis. Radiat Oncol 2020;15(1):128.

8. Milano MT, Grimm J, Niemierko A, Soltys SG, Moiseenko V, Redmond KJ, et al. Single-and multifraction stereotactic radiosurgery dose/volume tolerances of the brain. Int J Radiat Oncol Biol Phys 2021;110:68-86.

9. Hofmaier J, Bodensohn R, Garny S, Hadi I, Fleischmann DF, Eder M, et al. Single isocenter stereotactic radiosurgery for patients with multiple brain metastases: Dosimetric comparison of VMAT and a dedicated DCAT planning tool. Radiat Oncol 2019;14(1):103.

10. Moon YM, Jeon W, Yu T, Bae SI, Kim JY, Kang JK, et al. Which is better for liver SBRT: Dosimetric comparison between DCAT and VMAT for liver tumors. Front Oncol 2020;10:1170.

11. Clements M, Schupp N, Tattersall M, Brown A, Larson R. Monaco treatment planning system tools and optimization processes. Med Dosim 2018;43(2):106-17.

12. Bodensohn R, Kaempfel AL, Fleischmann DF, Hadi I, Hofmaier J, Garny S, et al. Simultaneous stereotactic radiosurgery of multiple brain metastases using single-isocenter dynamic conformal arc therapy: A prospective monocentric registry trial. Strahlenther Onkol 2021;197(7):601-13.

13. Eekers DB, In't Ven L, Roelofs E, Postma A, Alapetite C, Burnet NG, et al. The EPTN consensus-based atlas for CT-and MR-based contouring in neuro-oncology. Radiother Oncol 2018;128(1):37-43.

14. Benedict SH, Yenice KM, Followill D, Galvin JM, Hinson W, Kavanagh B, et al. Stereotactic body radiation 
therapy: The report of AAPM task group 101. Med Phys 2010;37(8):4078-101.

15. Paddick I, Lippitz B. A simple dose gradient measurement tool to complement the conformity index. J Neurosurg 2006;105:194-201.

16. Shaw E, Kline R, Gillin M, Souhami L, Hirschfeld A, Dinapoli R, et al. Radiation therapy oncology group: Radiosurgery quality assurance guidelines. Int $\mathrm{J}$ Radiat Oncol Biol Phys 1993;27(5):1231-9.

17. Audet C, Poffenbarger BA, Chang P, Jackson PS, Lundahl RE, Ryu SI, et al. Evaluation of volumetric modulated arc therapy for cranial radiosurgery using multiple noncoplanar arcs. Med Phys 2011;38(11):5863-72.

18. Molinier J, Kerr C, Simeon S, Ailleres N, Charissoux $\mathrm{M}$, Azria D, et al. Comparison of volumetric-modulated arc therapy and dynamic conformal arc treatment planning for cranial stereotactic radiosurgery. J Appl Clin Med Phys 2016;17(1):92-101.
19. Salkeld AL, Unicomb K, Hayden AJ, van Tilburg K, Yau S, Tiver K. Dosimetric comparison of volumetric modulated arc therapy and linear accelerator-based radiosurgery for the treatment of one to four brain metastases. J Med Imaging Radiat Oncol 2014;58(6):722-8.

20. Huang C, Ren L, Kirkpatrick J, Wang Z. SU-E-T-645: Treatment of multiple brain metastases using stereotactic radiosurgery with single-isocenter volumetric modulated arc therapy: Comparison with conventional dynamic conformal arc and static beam stereotactic radiosurgery. Med Phys 2012;39(20):3854.

21. Badkul RK, Jiang H, Saleh H, Chen AM, Wang F. Dosimetric evaluation of automated single-isocenteric vs. conventional multi-isocenteric SRS technique using dynamic conformal arcs for the treatment of multiple brain metastasis. Int J Radiat Oncol Biol Phys 2018;102(3):e506-7. 